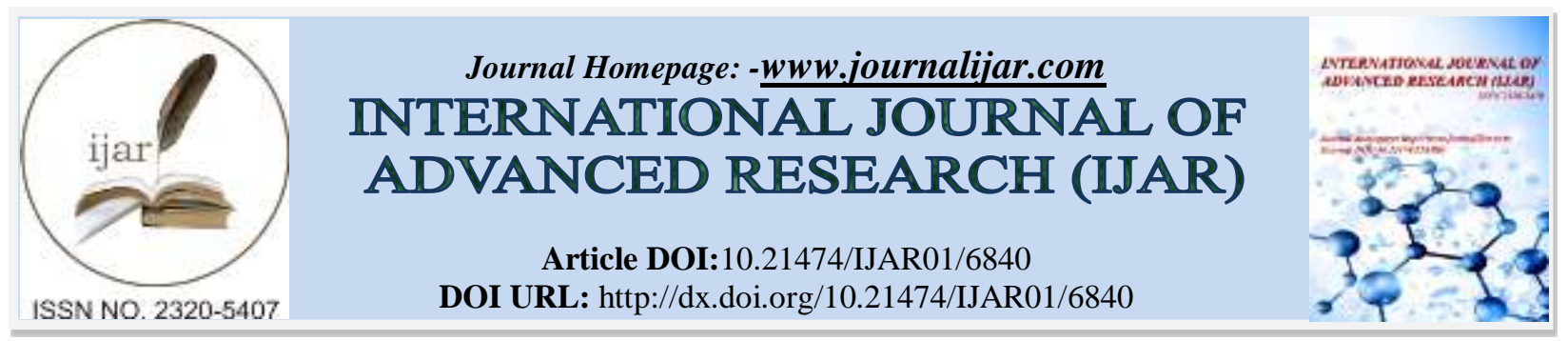

RESEARCH ARTICLE

\title{
ERREURS INTERFÉRENTIELLES ET DISPOSITIFS D'APPRENTISSAGE : CAS DES LYCÉENS TACHELHITOPHONES.
}

Tarnaoui Moulay Mohamed.

Université IBN Zohr- Agadir Maroc.

\section{Manuscript Info}

Manuscript History

Received: 04 February 2018

Final Accepted: 06 March 2018

Published: April 2018

Keywords:-

Erreur Interférentielle- Enseignement/ Apprentissage - Français Langue Etrangère - Langue Maternelle (Tachelhit) -Stratégie d'apprentissage.

\begin{abstract}
This study is part of a didactic and linguistic perspective. It aims to analyze the interferential deviations of Tachelhit students in the region of Agadir Ida_Outanane that abound in the production of learners despite the reforms. We asked the learners to write letters and from this support we collected the data. The choice of the support is justified by the motivation of the pupils to the epistolary communication. Moreover, it is one of the language needs of students in an era where plurilingualism is an asset and no longer a handicap. In the context of this work, we have apprehended interference (IF) as a learning strategy and not an index of bad that inhibits or blocks the act of appropriation. The results of the categories of analysis (morphology, syntax and lexicon) and the variables taken into consideration (sex, age and place of study) revealed the possibility of using interference as a learning strategy to optimize teaching. : learning.
\end{abstract}

Copy Right, IJAR, 2018,. All rights reserved.

\section{Introduction:-}

La didactique des langues étrangères (dorénavant DLE) traverse une phase importante de son histoire. Sa constitution et sa légitimation scientifiques passent par une interrogation historique, une auto-analyse en termes de champ, une institutionnalisation diversifiée, mais d'abord par une délimitation et une construction problématisée de son domaine et de ses objets (Coste ,1982; Besse et Porquier ,1980). Les recherches sur l'acquisition des langues étrangères sont susceptibles d'apporter des éclairages nouveaux sur le procès d'enseignement /apprentissage qui est au cour de la DLE. Elles peuvent susciter de nouveaux chantiers dans ce dernier domaine autour des profils d'apprenants, de la structuration de la matière à enseigner ou encore de l'évaluation (Martinez, 2008).

Ce qui caractérise ce champ didactique, c'est l'aspect de la controverse et de la dynamique. Pour Besse (1987 ; Besse et Galisson 1980), la didactique est un domaine interdisciplinaire à la recherche d'une relative autonomie scientifique. Bronckart (1989), quant à lui, met en œuvre le processus qui prend en charge l'observation, l'analyse et les décisions : il s'agit en somme d'un raisonnement expérimental. Galisson $(1989,1990)$ synthétise l'évolution de cette discipline qu'il nomme " didactologie/didactique des langues et des cultures". Le chercheur doit prendre en considération un certain nombre d'interrogations qui balisent le champ de la recherche didactique, à savoir pourquoi enseigner /apprendre? Comment ? Qui ?A qui ? Quoi enseigner/apprendre? Quand et où ? Pour Puren (1999, 2008), la didactique des langues est arrivée à une étape de son évolution historique où elle fonctionne à la fois comme méthodologie, didactique et didactologie des langues-cultures. Il est bien sûr possible - et c'est même 
l'approche la plus souhaitable parce que la plus adaptée à la complexité de son objet et de son domaine, de mettre simultanément en relation les trois niveaux.

Dans le cadre de cet article, la communication épistolaire sera, pour nous, un support et un appui considérables, du moment que tout un chacun, le public scolaire marocain en l'occurrence, a besoin d'écrire, à un moment ou un autre de sa vie, des lettres aux prochains, aux amis ...etc. Ainsi, nous servons des lettres amicales écrites par des élèves tachelhitophones pour la collecte de nos données. Notons que l'apprentissage de la rédaction d'un type d'écrit passe par la mise en évidence de sa spécificité à travers des caractéristiques de genre.

Le support épistolaire permet à l'apprenant d'exprimer librement ses idées, ses sentiments, sa conception des choses et du monde. Dans cette étude, nous avons essayé de localiser et d'analyser l'IF entre le tachelhit et le FLE des lycéens marocains du niveau tronc commun (TC), 1ème année et 2ème année Lettres Modernes(LM) appartenant à deux Délégations du Sud, à savoir Agadir-Ida Outanan et Chtouka-Ait Baha. Pour réaliser ce travail, nous avons combiné deux techniques: l'analyse contrastive (dorénavant AC) et l'analyse des erreurs (dorénavant AE).

Le problème qui fait l'objet de l'actuelle recherche est les déviations interférentielles commises par les apprenants tachlhitophones en FLE dans un milieu institutionnel marocain. Dans cette optique, notre étude vise la liaison de deux aspects :

L'aspect théorique d'une notion déjà débattue par les chercheurs, mais elle demeure toujours vivace dans le domaine de l'enseignement / apprentissage. Il s'agit de l'IF linguistique qui persiste dans l'écrit et dans l'oral des élèves. La confrontation des données linguistiques et les pratiques du FLE peuvent confirmer ou infirmer les difficultés rencontrées par les élèves marocains dans l'appropriation de cette langue étrangère. Dans le cadre de ce travail, nous avons opté pour la LM des apprenants, le tachelhit -parler de la région d'Agadir et le FLE.

L'aspect pratique et ceci va s'effectuer à travers l'analyse du FLE. Dans le cadre de cette étude, il sera question de procéder à une étude des interférences des élèves tachelhitophones apprenant le FLE.

Ainsi, notre premier objectif consiste à relever les IF dans les lettres des élèves pour savoir si ces dernières entravent l'apprentissage du FLE et par conséquent des solutions seront nécessaires à ce problème. Le second objectif de cette étude est donc de chercher dans quelle mesure l'IF peut-elle devenir un moyen d'apprentissage? Nous rappelons qu'employer une stratégie c'est agir pour atteindre un but déterminé. Le terme " stratégie » suggère que l'apprenant choisit consciemment les moyens jugés les plus efficaces pour accomplir sa tâche. Ces stratégies sont présentées comme des caractéristiques individuelles de l'apprenant .Dans cette veine, il est légitime de poser les questions suivantes: Comment permettre aux apprenants de se constituer progressivement des stratégies qu'ils useront en situation de production? Comment fonctionne la mémoire de l'apprenant dans le fonctionnement de l'information? Comment créer un climat de confiance pour que les élèves puissent s'accommoder des contraintes de l'écrit ? (Cornaire, 2014).

Nous essayons de cerner la notion d'interférence sur le plan didactique qui nous préoccupe. Nous rappelons que l'interférence est apparue avec l'analyse systématique de la performance des apprenants de LE. C'est le processus qui aboutit à la présence dans un système linguistique donné d'unités et souvent d'agencement appartenant à un autre système.

Lado (1957) nous parle d'interférence qui affecte la phonétique, la grammaire, le lexique et la sémantique mais aussi l'écriture et la culture. Les erreurs d'apprentissage sont prévisibles et prédites grâce à l'analyse contrastive des langues.

Sur le plan didactique, il s'agit de mettre le doigt sur les difficultés rencontrées par l'élève et les erreurs qu'il commet en LE du fait de l'influence de la LM ou d'une LE étudiée antérieurement (Coste et Galisson, 1976).

Mackey (1982), quant à lui, saisit ce phénomène à l'étude comme l'utilisation des éléments d'une langue dans le discours d'une autre langue. En outre, il conçoit l'interférence comme un phénomène du discours.

En tenant compte de ces définition, nous voulons savoir comment l'IF pourrait devenir une stratégie utilisable dans l'enseignement / apprentissage. L'évolution de la DLE a montré que l'erreur est un indice positif d'apprentissage 
(Larry 2014 ; Lokman Demirtaş et Hüseyin Gümüş, 2009). L'IF en tant qu'erreur provenant d'une LM ou d'une autre langue s'inscrit dans cette perspective.

A la suite de Corder (1967), beaucoup de chercheurs se remettent à la recherche des erreurs en faisant la nécessaire distinction entre les erreurs interférentielles et celles qui n'avaient pas leur origine dans la LM des apprenants. Les chercheurs arrivent à sérier et à interpréter une partie d'entre elles comme indépendantes des structures linguistiques de LE (Jamet, 2000 ; Svetlana Tyaglova , 2008 ; Boonprome, 2009a ; Boonprome, 2009b ).

De là ressortent les suppositions qui balisent notre champ d'investigation. Nous voulons vérifier une hypothèse de base : L'IF en tant que stratégie pourrait être utilisée pour optimaliser un enseignement / apprentissage en milieu institutionnel marocain. Dans le cadre de cette étude, nous allons essayer de confirmer ou d'infirmer cette réponse provisoire supposée qui constitue le noyau de la recherche.

Dans la perspective où l'IF est appréhendée en tant que stratégie d'apprendre, dans quelle mesure peut -on concevoir des outils pédagogiques pour optimaliser l'enseignement / apprentissage ? En outre, il est capital de poser la question suivante : d'où viennent les IF des élèves tachelhitophones?

\section{Résultats Et Discussion:-}

La distribution des résultats d'après les catégories d'analyse s'avère pertinente en morphologie et en syntaxe du moment que ces deux catégories sont les plus affectées par les déviations interférentielles. Cela reste vrai pour la 1 AS, la 2 AS et la 3 AS du lycée, section : lettres modernes.

En ce qui concerne la morphologie qui occupe la première place, il paraît que l'influence de la LM - Le tachelhit est certaine. Cette catégorie d'analyse représente $52.9 \%$ en 1 AS, $55 \%$ en 2 AS et $47 \%$ en 3 AS. Dire que le tachelhit est le seul médium responsable de ces constructions déviantes est hasardeux. En effet, d'autres facteurs interviennent avec force comme l'arabe marocain et l'arabe moderne; il s'agit des systèmes linguistiques connus, emmagasinés des sujets.

Les IF syntaxiques peuvent affecter les différents niveaux d'organisation du langage. Dans cette optique, les interférences syntaxiques sont saisies comme des déviations qui touchent la structure de l'énoncé, l'ordre des mots (Coste et Galisson, 1976; Martinet, 1969). Nous avons opté pour l'analyse des prépositions, de la conjonction copulative "et" et l'ordre des mots.

Il s'agit à cet effet d'étudier les règles qui président à l'ordre des mots et à la construction des phrases en FLE par des sujets tachelhitophones. Des recherches sont entreprises dans le cadre de la syntaxe comme celle de Debysser (1970), Saad (1992), Hammami(1992), Afari(2012),Veyrenc(2015).

Notre corpus démontre que la syntaxe occupe une place considérable. Les constructions déviantes constituent $36 \%$ en $1 \mathrm{AS}, 40 \%$ en 2 AS et $44 \%$ en 3 AS. Les erreurs sur les prépositions viennent en première position. Nous avons localisé 166 déviations en 1 AS, 94 en 2 AS et 53 en 3 AS. Dans le cadre de la syntaxe, nous signalons que c'est le cas le plus complexe. C'est une difficulté principale pour les apprenants; d'où la fréquence des transferts négatifs qui altèrent la structure de l'énoncé.

Dans cette optique, les IF dues aux prépositions viennent d'un choix inadéquat d'éléments qui pourrait être imputé à l'IF des langues stockées (tachelhit, l'arabe marocain, l'arabe moderne). Ces résultats rejoignent partiellement ceux d'autres études où les erreurs les plus typiques dénotent les déficiences caractéristiques dans l'appropriation des mécanismes du français pour les arabophones se situent au niveau des prépositions et de la concordance des temps.

Dans le même sillage, Chami (1987) souligne que le système prépositionnel ne fonctionne pas de la même manière en arabe et en français. Les verbes transitifs indirects en français ne correspondent pas aux verbes transitifs indirects en arabe. Ainsi, l'enseignant doit recourir aux systèmes prépositionnels de la LM des élèves pour fins de comparaison avec le système prépositionnel du FLE. La visée est de les stimuler à être plus prudents, avisés dans le choix convenable de la préposition. 
Vouloir limiter l'étude de la préposition à la classe grammaticale des prépositions, c'est ignorer son statut. Cette particule ne dépend pas uniquement d'une partie du discours. Parler d'erreurs concernant les prépositions, c'est évoquer la langue toute entière, pour la bonne et la simple raison qu'à chaque fois qu'on parle d'expansion ou de constituants supplémentaires, la préposition se trouve impliquée. On ne pourra jamais la dissocier de l'ensemble de la langue. Vouloir limiter le traitement automatique de l'erreur à une rectification automatique de la préposition est une technique vouée à l'échec. Avant de penser au traitement didactique, le professeur devrait chercher l'origine de la déviation.

Le second élément à étudier dans le cadre de la syntaxe est l'ordre des mots. Nous avons recensé 73 déviations en $1 \mathrm{AS}, 58 \mathrm{en} 2 \mathrm{AS}$ et 28 en $3 \mathrm{AS}$. Par conséquent, l'ordre des mots pose des problèmes. Les perturbations dans l'ordre de la phrase sont importantes puisque «la non - maturation syntaxique » persiste. La place des constituants de la phrase est intéressante à étudier. L'ordre des énoncés de nos sujets tachelhitophones est claqué sur la forme de tachelhit. L’ordre le plus usité en tachelhit est V.S.O. (verbe, sujet, objet) par contre celui du français reste S.V.O.

Nolke (1996) se demande dans un article «Où placer l'adjectif épithète ? ». Sa réflexion est constructive à cet égard : comment se fait-il que l'adjectif tantôt précède son substantif et tantôt lui succède ? Tantôt le même adjectif accepte les deux positions sans notable distinction de sens, tantôt l'antéposition et la postposition correspondent à deux valeurs nettement différentes. Dans d'autres cas encore, seule une des positions est possible. Nous citons dans le même cadre une étude menée par Afari (2012) qui a décrit le caractère de l'IF linguistique de l'anglais qui détermine la spécificité des fautes chez les étudiants apprenant une LE. C'est le transfert négatif qui entrave et achoppe l'apprentissage. Les catégories d'analyse prises en considération sont les pronoms, les accents, les adjectifs.

Pour la conjonction de coordination «et», elle pose à son tour des difficultés qui ne sont pas considérables par rapport aux prépositions et à l'ordre des mots. Le repérage est de 17 énoncés déviants en $1 \mathrm{AS}, 19$ en $2 \mathrm{AS}$ et 13 en 3AS. Cependant le traitement didactique de ces constructions est facile. Ainsi, en français, on sépare chaque terme par une virgule, et on n'emploie la conjonction copulative « et » qu'avant le dernier terme de l'énumération. En tachelhit, la virgule n'existe pas et on répète le [d] - équivalent de «et » en français - avant chaque terme de l'énumération. Cependant la répétition [d] n'est pas systématique. Les deux énoncés existent : [bba d inna dxali d gwma] et [bba inna xali d gwma ${ }^{1}$.

Les trois éléments pris dans le cadre de la syntaxe, à savoir les prépositions, l'ordre des mots et la conjonction copulative montrent que la composante syntaxique se révèle assez complexe étant donné qu'elle possède des liens multiples avec les composantes sémantiques et lexicales. Ce qu'une langue exprime par exemple par des moyens syntaxiques est exprimé avec des moyens lexicaux dans une autre langue ou ce qui est exprimé par un verbe dans une langue est rendu par un nom dans une autre.

Du point de vue statistique, la composante lexicale est en troisième place avec 32 déviations repérées en $1 \mathrm{AS}, 20$ en $2 \mathrm{AS}$ et 20 en $3 \mathrm{AS}$. Mais d'un point de vue pédagogique, la réflexion sur la nécessité de rendre sa place à l'étude du vocabulaire est capitale. Les IF lexicales sont assez importantes du point de vue de leur fréquence dans le corpus du moment qu'elles démontrent que le système lexical chez l'élève souffre de lexique précis et approprié.

Dans cette perspective, Galisson (1983 :.26) soutient que «l'accès à l'usage et au sens des mots est d'abord et surtout un problème d'organisation et de mémorisation ». Il est à noter que l'emprunt et le calque sont les formes les plus caractéristiques des déviations lexicales. Un autre type de difficultés intervient lorsque le français fait une distinction que le tachelhit ignore à titre d'exemple connaître / savoir, casser / briser / rompre, couper / traverser / diviser, attraper / recevoir...etc. Pour compenser ces failles, l'enseignement du vocabulaire devient prioritaire. L'élaboration des exercices relatifs à l'étymologie, à la synonymie, à la polysémie, à l'homonymie....etc. seront opératoires. Dans ce sens, la corrélation entre la syntaxe et la sémantique est pertinente. Cette réciprocité mérite d'être approfondie.

\footnotetext{
${ }^{1}$ [mon père et ma mère et mon oncle et mon frère]
} 
Des études sont réalisées dans ce domaine. Certains auteurs abordent les approches lexicologiques précisent que la lexicométrie se fonde sur l'exhaustivité des relevés, l'uniformité du dépouillement, l'unicité du critère de dépouillement. Nous militerons pour une réponse claire à la question: quelle place donner aujourd'hui au vocabulaire dans l'enseignement / apprentissage ? La maîtrise d'une LM ou d'une LE passe nécessairement par la maîtrise des mots. La forte demande des apprenants en matière de vocabulaire prouve suffisamment que les mots restent bien utiles pour communiquer.

\section{Conclusion:-}

Nous avons constaté que la majorité des erreurs interférentielles peut être attribuée à la langue maternelle des apprenants. En outre, les règles élémentaires de base ne sont pas maitrisées. A défaut d'une compétence linguistique ajustée à leur besoin, il recourt à L1 pour suppléer le manque, c'est un stade évolutif de l'apprentissage (interlangue). Les problèmes du FLE émergent de l'enseignement fondamental; d'où le rejet ou l'immotivation des enfants et par la suite les collégiens et les lycéens. Les élèves souffrent réellement d'un véritable handicap linguistique. Ainsi comment enseigner les autres composantes de la compétence interculturelle (composante ethnolinguistique, actionnelle, relationnelle, interprétative et interculturelle sachant pertinemment que les apprenants ne disposent pas d'une composante linguistique Beacco (2007)?

Il ressort de cette étude qu'effectivement on pourra employer l'IF comme stratégie d'apprentissage. Dans cette optique, l'exploitation des grilles didactiques est opératoire. Ainsi, l'hypothèse de base est validée. Il sera impérieux que l'expérimentation didactique, mette en œuvre la réactivation des mécanismes d'enseignement / apprentissage du FLE en focalisant l'attention sur les besoins de l'apprenant dans une époque où le plurilinguisme est un atout et non plus un handicap.

\section{References Bibliographiques:-}

1. Afari, E. (2012) : Réflexion sur l'interférence ou le transfert linguistique de l'anglais sur la production orale et écrite des étudiants du department de français à UEW Wirneba: Enjeux et Perspectives .Eureka-Unilag, a journal of Humanistic Studies, 2.

2. Beacco J.-C. (2007) : L'approche par compétences dans l'enseignement des langues. Enseigner à partir du Cadre européen commun de référence pour les langues, Didier.

3. Besse, H.,Porquier..(1980) : Grammaire et didactique des langues. Paris, CREDIF, LAL, Hatier

4. Besse, H.,Galisson,R. (1980) : Polémique en didactique.Paris, CLE International.

5. Boonprome, J. (2009a): The error system in French pronunciation by Thai learners (Rapport de recherche).Chiangmai:ChiangmaiUniversity.

Boonprome, J. (2009b) : Evaluation of the effectiveness of phonetic correcting strategies for the Improvement of French pronunciation ability by Thai students. Prosodies, vocalic sounds and consonant sounds. Songklanakarin Journal of Social Sciences and Humanities, 15(6), 1037-1056.

6. Chami, M. (1987) : L'enseignement du français au Maroc : diagnostic des difficultés et implications didactiques. Casablanca, Najah el Jadida.

7. Cornaire, Claudette (2014) : La production écrite. Paris, Clé International.

8. Coste, D. (1982) : Didactique et diffusion du FLE : questions de priorité. Etudes de Linguistique Appliquée, 64.

9. Coste, D., Galisson, R. (1976) : Dictionnaire et didactique des langues. Paris, Hachette.

10. Coulibaly, B. (1994) : Interférences et français populaire du Burkina. Langue Française, 104.

11. Debysser, F. (1970) : La linguistique contrastive et les interférences. Langue Française, 8.

12. Demirtaş L. et Hüseyin Gümüş. (2009) : De la faute à l'erreur: une pédagogie alternative pour améliorer la production écrite en FLE. Synergies Turquie ${ }^{\circ} 2$ - 2009 pp. 125-138.

13. Galisson, R. (1980 b) : Préambule. Lignes de force du renouveau actuel en didactique des langues étrangères. Paris, CREDIF, LAL, Hatier.

14. Galisson, R. (1982) :D'autres voies pour la didactique des langues. Paris, CREDIF, LAL, Hatier.

15. Galisson, R. (1983) : Des mots pour communiquer : éléments de lexicométhodologie. Paris, CLE International.

16. Galisson, R. (1989) : Enseignement et apprentissage des langues et des cultures, «évolution » ou « révolution » pour demain ? Le Français dans le Monde, 227.

17. Jamet, C. (2000) : Contrastivité et enseignement du français langue étrangère en France : approche anthropodidactique (Thèse de doctorat publié). Université de Lyon 2, France.

18. Galisson, R. (1990) : Où va la didactique du français langue étrangère ? Etudes de Linguistique Appliquée, 79. Larruy, Marquillo. 2014 : L'interprétation de l'erreur .Paris, Clé International 
19. Mackey, W. (1982) : Interaction, interférence et interlangue : Rapport entre bilinguisme et didactique des langues. Langues et linguistique, tome 1, Université Laval.

20. Maingueneau, D. et E. Pellet.(2005): Les notions grammaticales au collège et au lycée, Guide Belin de l'enseignement. Paris, Belin

21. Martinez,P.(2008) : La didactique des langues étrangère Paris, PUF. Collection : Que sais-je ?

22. Nolke, H. (1996) : Où placer l'adjectif épithète : focalisation et modularité. Langue Française, 111.

23. Puren, Christian (1999) : "La didactique des langues-cultures étrangères entre méthodologie et didactologie ", Les langues Modernes n 3 , Paris APLV, pp.26-4

24. Puren, Christian(2008) : La didactique des langues étrangères à la croisée des méthodes: Essai sur l'éclectisme 3ème édition, www.christianpuren.com,octobre 2013

25. Tyaglova Svetlana. (2008) : Analyse psycholinguistique des écarts `a la norme discursive. Structures du français en contact, Jun 2008, Nouvelle-Orléans, United States. pp.8. <halshs-00317652>

26. Wende, O. (2014). Interférence linguistique chez les francissants anglophones : le cas du présent de l'indicatif. International Journal of English Language and Linguistics Research Vol.2, No. 1, pp. 31-38, March 2014 Published by European Centre for Research Training and Training and Development UK.

27. J. Veyrenc. (1968) : Interférences syntaxiques et ordre des mots en russe, Word, 24:1-3, 498-507, DOI:10.1080/00437956.1968.11435551. Publié online le 16 Juin 2015. 